\title{
Packing, entropic patchiness, and self-assembly of non- convex colloidal particles: a simulation perspective
}

DOI:

10.1016/j.cocis.2017.05.005

\section{Document Version}

Accepted author manuscript

Link to publication record in Manchester Research Explorer

\section{Citation for published version (APA):}

Avendano, C., \& Escobedo, F. A. (2017). Packing, entropic patchiness, and self-assembly of non-convex colloidal particles: a simulation perspective. Current Opinion in Colloid and Interface Science.

https://doi.org/10.1016/j.cocis.2017.05.005

\section{Published in:}

Current Opinion in Colloid and Interface Science

\section{Citing this paper}

Please note that where the full-text provided on Manchester Research Explorer is the Author Accepted Manuscript or Proof version this may differ from the final Published version. If citing, it is advised that you check and use the publisher's definitive version.

\section{General rights}

Copyright and moral rights for the publications made accessible in the Research Explorer are retained by the authors and/or other copyright owners and it is a condition of accessing publications that users recognise and abide by the legal requirements associated with these rights.

\section{Takedown policy}

If you believe that this document breaches copyright please refer to the University of Manchester's Takedown Procedures [http://man.ac.uk/04Y6Bo] or contact uml.scholarlycommunications@manchester.ac.uk providing relevant details, so we can investigate your claim.

\section{OPEN ACCESS}




\section{Accepted Manuscript}

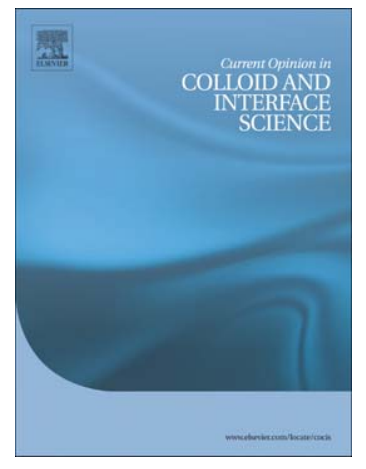

Packing, entropic patchiness, and self-assembly of non-convex colloidal particles: a simulation perspective

Carlos Avendaño, Fernando A. Escobedo

PII:

S1359-0294(17)30049-3

DOI: doi: $10.1016 /$ j.cocis.2017.05.005

Reference: $\quad$ COCIS 1122

To appear in: $\quad$ Current Opinion in Colloid \& Interface Science

Received date: $\quad 24$ March 2017

Revised date: 21 May 2017

Accepted date: 23 May 2017

Please cite this article as: Avendaño Carlos, Escobedo Fernando A., Packing, entropic patchiness, and self-assembly of non-convex colloidal particles: a simulation perspective, Current Opinion in Colloid \& Interface Science (2017), doi:10.1016/j.cocis.2017.05.005

This is a PDF file of an unedited manuscript that has been accepted for publication. As a service to our customers we are providing this early version of the manuscript. The manuscript will undergo copyediting, typesetting, and review of the resulting proof before it is published in its final form. Please note that during the production process errors may be discovered which could affect the content, and all legal disclaimers that apply to the journal pertain. 


\title{
Packing, entropic patchiness, and self-assembly of non-convex colloidal particles: a simulation perspective
}

\author{
Carlos Avendaño ${ }^{1, a)}$ and Fernando A. Escobedo ${ }^{2, b)}$ \\ 1) School Chemical Engineering and Analytical Science, The University of Manchester, Sackville street, Manchester, \\ M3 9PL, UK \\ ${ }^{2)}$ School Chemical iand Biomolecular Engineering, Cornell University, Ithaca, NY, 14853, \\ USA
}

Recent advances in experimental techniques to synthesise particles with non-convex shapes have provided new challenges and opportunities to the modelling community. The availability of such building blocks has motivated many computational studies on the formation of new materials driven by such complex effects as interpenetration, interlocking, and shape complementarity that, if properly harnessed, have the potential of acting as entropic directional (patchy) interactions in particles with non-convex geometries. This article highlights recent molecular simulation studies of anisotropic non-convex colloidal particles with particular emphasis in particles interacting via excluded volume.

\section{SELF-ASSEMBLY IN COLLOIDAL SYSTEMS}

The shape of colloidal particles is one of the most important features that dictates their complex collective behavior. As a result of recent advances in experimental techniques that allow the synthesis of colloidal particles with arbitrary shape and size $\mathrm{e}^{1-4}$, it is currently possible to use these particles as building blocks for the selfassembly of complex structures. Colloidal particles are appealing not only for fundamental studies as they are often large enough to allow direct observation of their self-organisation and dynamics, but also for applications as it is their size what makes them suitable candidates for the fabrication of mesoscopic structures that are not easily attainable using molecular systems ${ }^{5,6}$. Furthermore, the ability to tune the repulsive and attractive contributions of the interparticle interactions by changing the properties of both the particle surface (surface charge, functional groups, tethered ligands, etc.) and the solvent medium has made possible an unprecedented level of control of complex structural designs ${ }^{7-10}$.

In the absence of significant attractive interactions, the properties of colloidal suspensions are mainly determined by packing effects that tend to increase the particles' translational entropy, thereby decreasing their free energy ${ }^{11}$. Computer simulations of hard-core particles, i.e., particles interacting purely by steric interactions, have played a central role in understanding the structure and order-disorder transitions of colloidal systems. ${ }^{12} \mathrm{Nu}-$ merous simulation studies have been reported focusing on the behavior of spherical particles and many other convex-shaped geometries, including unusual shapes like polyhedra ${ }^{13-15}$. For certain faceted convex shapes, the concept of entropic patchiness has been introduced to described the entropic directional forces taking place in the systems even in the absence of attractive interactions that promotes the formation of ordered faces.

\footnotetext{
a) Electronic mail: carlos.avendano@manchester.ac.uk

b) Electronic mail: fe13@cornell.edu
}

These forces emerge from the collective behaviour of the colloidal systems at sufficiently high concentrations ${ }^{15,16}$ Colloidal particles with non-convex geometries, however, have received far less attention despite the significant advances that have been made in experimental techniques for their fabrication. Some examples of non-convex particles available with existing experimental techniques are shown in Figure 1. A particle is said to be non-convex if, there exist two points inside the particle such that the line segment connecting them does not lie entirely inside the particle. This geometrical representation of non-convex particles is shown in Figure 1(a). Different descriptors can be used to characterise the degree of anisotropy of the particles such as aspect ratio, circumscribed sphere radius, isoperimetric quotient, etc. ${ }^{16-18}$ It is also useful to adopt a metric to describe the degree of convexity $\zeta$ of particles having arbitrary shape. One such choice, sketched in Figure 1(b), is to define $\zeta$ as the ratio of the volume of a particle $V_{\mathrm{p}}$ to the volume of its convex envelop (convex hull) $V_{\mathrm{ch}}$, i.e.,

$$
\zeta=\frac{V_{\mathrm{p}}}{V_{\mathrm{ch}}}
$$

Note that in the limit of $\zeta \rightarrow 1$ a particle becomes convex, while $\zeta \rightarrow 0$ indicates high degree of non-convexity. Alternatively, one could identify shape-complementary patches in the particles, e.g., by the convex and nonconvex regions of two particles that fit closely together (akin to entropic lock-and-key or patchy attractions, see Figure 1(c)). Some of the earlier examples of non-convex shapes investigated were slight variants of their convex counterparts, such as the case of "banana"-shaped particles shown in Figure $1(\mathrm{~d})^{19,20}$, whose phase behavior provided interesting departures to the known liquidcrystalline phases of rods and spherocylinders. Arguably, polymers, which have been extensively studied and are often modelled as bead-spring chains, can be regarded as "flexible" or re-configurable non-convex Brownian objects. Although some of the peculiar characteristics of polymer-polymer interactions (like the tendency to form entanglements) are ubiquitous in non-convex shapes, this 
review is only concerned with "rigid" particles that lack conformational entropy; however, small oligomers and dimers in particular constitute a natural point of intersection and divergence between those two classes of materials.

In general, single-component non-convex particles do not pack very efficiently since they are characterised by a free volume within their convex hull that is often inaccessible to neighbouring particles. This is particularly evident when the degree of convexity $\zeta \rightarrow 0$ such as in the case of thin rings (see Figure $1(\mathrm{e}))^{21,22}$ and branched colloids ${ }^{23}$ (see Figure 1(f)). Inefficient packing in this context, however, does not always imply disordered states. In fact, several studies have shown that highly symmetric non-convex particles can form ordered structures having high free volumes, i.e., porous ordered materials $^{22-25}$.

Non-convex particles offer many possibilities for the fabrication of functional materials as a result of their ability to self assemble into unique structures driven by mechanisms absent in convex particles such as particle interlocking, entanglement, and interpenetration ${ }^{21,24-29}$. These emerging materials are thus promising in a broad range of applications including drug delivery and

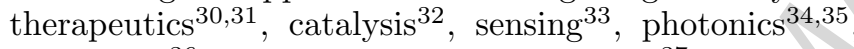
absorption $^{36}$ and nanopattern scaffolding ${ }^{37}$. From a modelling perspective, NC particles represent multiple challenges. For instance, global ergodic equilibration can be compromised by the occurrence of local interlocking configurations. Furthermore, in most cases, the particle geometry and the interparticle hard-core repulsion cannot be represented via a simple analytical form. With some exceptions, most recent attempts to model non-convex particles involve outlining a shape by an ensemble of smaller convex objects. For example, branched colloids have be modelled as ensembles of rodlike particles ${ }^{38}$, rings have been modelled as rigid ensembles of spheres ${ }^{22,29,39}$, and even more complicated geometries have been represented using triangular tessellation methods ${ }^{24}$. While generally effective and adaptable, such "multi-site" particle models tend to be computationally more expensive than those that can describe particle interactions via a single mathematical function.

FIG. 1. Geometrical representation of (a) convex and nonconvex objects and (b) the convex hull envelope used to define the degree of convexity $\zeta$ of an object, exemplified here for a dimer and a cubic frame; (c) Example of a particle shape containing both convex and non-convex (patchy) section. Two particles are shown forming a lock-and-key bond. Examples of non-convex particle shapes that are accessible with current experimental techniques: (d) curved (banana shaped) and twisted particles, and circular segments ${ }^{39-41}$; (e) frames, rings, and cages $30,32,42-44$; (f) branched particles and non-convex polyhedra ${ }^{23,45-48} ;(\mathrm{g})$ dimers, clusters and protruded particles ${ }^{49,50}$; (h) bowls, lenses, indented and multicavity particles ${ }^{51-55}$.

The introduction of surface roughness to selected ar- eas of convex colloidal particles has been shown to be an effective means to induce directional interparticle attractions in the presence of depletants able to penetrate the roughness features ${ }^{56}$. In this manner, the smoother parts of different particles experience a depletion-induced attraction. In some respects, this non-trivial effect of roughness on interpaticle interactions is tied to the fact that introducing roughness also introduces a level of nonconvexity to the particle shape. While the length scale of those non-convex features is typically much smaller than the overall particle size (to warrant altering the shape's convex vs. non-convex taxonomy), it is comparable or large relative to the depletant size.

\section{HARD AND SOFT-CORE MODELS OF NON-CONVEX PARTICLES}

\section{A. Dumbbells, cluster-like and protruded particles}

Dumbbells, probably one of the simplest non-convex geometries, have attracted considerable attention due to their potential use for the fabrication of photonic materials ${ }^{34,57}$. A dumbbell can be seen as a dimer of two spheres and is hence comprised by two lobes of diameters $\sigma_{1}$ and $\sigma_{2}$ permanently bonded at a fixed bond length $l$. Despite the system being formed by simple spherical lobes, dumbbells with different degree of convexity exhibit the formation of unusual structures due to the additional orientational degrees of freedom (absent in single spheres). ${ }^{58-62}$ For example, computer simulations of hard homonuclear dimers, i.e., dumbbells formed by two tangent spheres with $\sigma=\sigma_{1}=\sigma_{2}$, are known to pack at high densities in a way similar to that of monodisperse spherical particles. However, the configurational degeneracy arising from the large number of possible arrangements of the particle bonds within the crystal lattice promotes the formation of aperiodic crystals ${ }^{63,64}$. This crystals have been observed in both two and three dimensional systems in the bulk ${ }^{58,65}$, and under strong geometrical confinement ${ }^{66}$. Dumbbells not only show a different phase behavior from spherical particles, but their phases also differ in mechanical and dynamical properties. The work of Gerbode and co-workers ${ }^{67,68}$, for example, demonstrates that peanut-shape particles in a $2 \mathrm{D}$ monolayer exhibit a unique and slow mechanism of dislocation dynamics arising from their higher proclivity to become "caged" than spheres, as the interlocking of neighboring particles tends to restrict particle mobility. Similar factors have also been observed in binary mixtures of dimers and spheres when subject to constant stress and constant shear rate, where the interplay between dislocations and vacancies give rise to complex rheological and mechanical behavior ${ }^{69}$. These factors lead to a non-trivial collective behavior that is not observed in simple spherical systems; e.g., a transitional phase occurs before complete shear-induced crystal melting where the rate of crystal growth (driven by thermodynamics) is 
balanced out by the rate of melting (driven by shear). Such an intermediate state exhibits shear banding at a local level, and a hexatic-like behavior at the macroscopic level. For dumbbells with different lobe diameters and bond lengths, computer simulation studies using Monte Carlo simulation and free energy calculations have shown that the particles can exhibit a very rich phase behavior ranging from plastic crystals to aperiodic crystals, and aligned crystals $61,62,70$

Changing the chemistry and the morphology (roughness) of one of the lobes of a dimer can also be used to target preferential interactions through the addition of depletants. This approach of controlling the interparticle interaction was demonstrated by Kraft and co-workers in a combined experimental and simulation study of asymmetric dimers comprised by one smooth lobe and one highly rough lobe ${ }^{71}$. The addition of depletant induces a net attractive interaction between the smooth lobes, as in janus particles ${ }^{72}$, leading to the formation of micellar aggregates. Experimentally, it is also possible to fabricate not only dimer-like particles but also trimers, multi spherical clusters ${ }^{49,55,73}$, colloidal molecules ${ }^{74}$, and particles with multiple patches in the form of protrusions decorating the surface ${ }^{71,75}$. Examples of these particle shapes are also shown in Figure $1(\mathrm{~g})$. The viability of these more complex, anisotropic particles has motivated various simulation studies to examine in detail the mechanism underlying their phase and crystallization behavior ${ }^{18,76}$, which could also help understand related phenomena in other complex systems such as in protein stability $^{77,78}$.

\section{B. Interlocking of highly non-convex particles: formation of superporous ordered materials}

In many instances, jamming, entanglement, and interlocking of anisotropic particles can lead to the formation of rigid disordered states ${ }^{79-82}$. It has been demonstrated, however, that these mechanisms can be harnessed for the assembly of porous ordered structures at the microscale. As interlocking in some symmetric nonconvex shapes can only take place at specific particle orientations, these interactions can be described as entropic directional forces, i.e. entropic patches. In particular, ring-shaped particles ${ }^{22}$ and octapods ${ }^{23}$ represent two outstanding examples in which particle interlocking is one of the main mechanisms responsible for the formation of porous ordered structures.

Frame-like particles are interesting systems that, depending on the materials used for their fabrication (metals, polymer, silica, DNA, etc.), have shown exceptional performance in various fields such as catalysis, electronics and plasmonics, and drug encapsulation and delivery ${ }^{32,83,84}$. Some examples of these particle shapes are shown in Figure 1(e). This performance is a consequence of the frames' intrinsic large surface to volume ratios, unusual optical properties, and significant
FIG. 2. Microstructures observed in circular colloidal rings with different aspect ratios. Particles with large cavities (a-c) exhibit the formation of smectic liquid crystalline phases (a layer of the smectic phase is also shown), while rings with small cavities (d) form disordered jammed phases. For each microstructure, two particles showing the maximum degree of interpenetration are also presented. Adapted from Ref. 22. Copyright 2016 National Academy of Sciences of the United States of America.

FIG. 3. Self-assembly mechanism of octapods in solution. This hierarchical process involves two steps: (a) the aggregation of the particles in linear chains, followed by (b) the arrangement of these linear chains side by side to form ordered superlatices. Adapted from Ref. 23. Copyright 2011 Macmillan Publishers Limited.

mechanical elasticity, coupled with chemical- or biocompatibility. The phase behavior and packing of colloidal rings, which can be seen as planar frame-like particles, has been studied recently using a soft-repulsive coarse-grained model ${ }^{22}$. This study demonstrated that the partial interpenetration between ring molecules can induce their interlocking and lead to the formation of layered structures, which assemble in the form of smectic liquid crystalline phases with intrinsic porosity $22,85,86$. In particular, it was shown that both the degree of interpenetration and the symmetry of the rings are the main factors determining the stability of the ordered phases: smectic phases are favoured in systems of particles with large, symmetrical internal cavities that are conducive to high degree of interpenetration. Representative configurations of microstructures formed by circular rings with various degrees of interpenetration are shown in Figure 2. The formation of porous smectic liquid crystalline phases observed in colloidal rings has also received a sound statistical mechanical basis using Onsager's theory at the second virial coefficient level ${ }^{87}$. The maximum packing of ring tori was also investigated by Gabbrielli et al. to show that major-to-minor radii ratios in the interval $[0$, 1.47074] allow maximum packing fractions, higher than that obtain with spherical objects, while ratios larger than 1.47074 lead to rapidly diminishing packing fractions due to the large internal cavity (thin rings) ${ }^{21}$.

Like nanoframes and nanorings, branched nanoparticles (nanocrystals) also possess high surface to volume ratios $^{88-90}$. These particles, which are shown in Figure $1(\mathrm{f})$, have been envisioned as potential building blocks for the fabrication of materials for solar cells and electronic devices ${ }^{88,91}$, and as systems suitable for biomedical applications such as tracers, drug delivery carriers, and as agents for photothermal cancer trament ${ }^{31}$. Recently, various techniques have been advanced to control the particle size and the length of the particle branches, thus opening the possibility to create particles that selfassemble into target ordered superlattices ${ }^{88}$. These techniques include, for example, the growth of arms from 
a crystal nucleus or the selective growth of secondary $\operatorname{arms}^{91}$. These events are driven by such mechanisms as polymorphism and twinning of crystals in the presence of a surfactant that can selectively attach to specific facets and promote the selective growth of arms ${ }^{89}$. One of the challenges associated with these particles lies in predicting and controlling their spatial organisation to target specific microstructures. Using a combination of experiments and computer simulations, Miszta and co-workers have studied the self-assembly of 8-arm branched particles (octapods) in solution ${ }^{23}$. Their results show that the formation of ordered superlattices from octapods is a two-step process if the solvent, that controls the interparticle interactions, is chosen appropriately. The first step of the process involves the formation of linear chains driven by the interlocking of a particles as shown in Figure 3(a), followed by the arrangements of the chains themselves to form a three-dimensional ordered porous superlattice (see Figure 3(b)). Further control of the structures formed by particles with different arm lengths can be achieved by confining the particles in monolayers ${ }^{38,46,92}$. This type of confinement is readily realized when a liquid droplet containing the octapods is placed on a substrate and allowed to rapidly evaporate. The shrinking of the droplet effectively confines the particles in a liquid-air quasi-two dimensional interface that gradually concentrate the system, promoting the ordering of the system. In fact, other studies of branched-like particles confined in two-dimensional monolayers have also shown the potential of non-convex particles to form unconventional structures. Experimental ${ }^{47}$ and simulation studies ${ }^{25}$, for example, have revealed the feasibility of fabricating chiral structures from the self-assembly of achiral cross-shaped particles confined in a plane, driven purely by the non-convex shape of the particles.

The large free volume attained in the structures from pure component systems of branched or frame-like colloids can potentially be occupied by a second component of appropriate shape and size. In such mixtures, the preferential contact between unlike particles implies that their interactions are effectively more attractive than those between like particles, a situation akin to of that of non-additive mixtures. This type of binary assembly, that resembles a jigsaw puzzle, was recently seen in mixtures of branched colloids with nanospheres ${ }^{93}$ and with nanoplates ${ }^{45}$. These two studies represent interesting examples of the concept of lock-and-key interaction, in which two components can shape complement to form ordered superlattices.

\section{Shape complementarity: Lock-and-key colloids}

We known by experience that bowls can be piled up to fit them in cabinets. We are also familiar with classical shape sorting toys used to help toddlers to develop spatial skills in which particular geometrical objects can only fit in specific cavities (shape complementarity). Similar fits are observed at the nano and microscales where the shape of non-centrosymmetric bowl-like and indented particles (see Figure 1(h)) can induce directional (patchy) interactions driven by entropic (packing) effects, giving rise to stacking, dimerization, and even (non-covalent) polymerization of the particles as shown in Figure $4^{53,54,100,101}$. Bowl-like particles can be synthesised from the buckling of thin spherical (hollow) shells. Experimental and simulation studies have shown that such hard colloidal bowls form worm-like randomly oriented stacks at sufficiently high concentrations, driven purely by entropic forces $^{54,102}$. A representative configuration of such phase is shown in Figure 4(c). Although reports on the staking of bowl-like molecules had been known before ${ }^{103,104}$, the work of Marechal and co-workers ${ }^{54}$ represented one of the first studies showing that entropy itself can drive the stacking of indented colloids at the mesoscale. When the indentation in the bowl becomes shallow, a different aggregation phenomenon is observed. For example, contact lens-like particles modelled as non-convex spherical caps, show multiple phases behavior, ranging from a liquid crystalline nematic phase to cluster-like aggregates ${ }^{105,106}$ depending on the curvature of the particles, with the aggregates being remarkably different to the structures observed in convex-spherical caps ${ }^{102,107-109}$.

With the aid of non-adsorbent polymers (depletant agents), the range and strength of these directional, interactions can be controlled even at low/moderate particle concentration to promote particle self-assembly, as demonstrated by experiments ${ }^{53,55,110}$ and by computer simulations $^{100}$. For example, the formation of worm-like colloidal polymers was demonstrated recently by Asthon and co-workers in a system of indented bowl-like colloids in the presence depletant particles (modelled explicitly as hard spheres) to induce attractive interactions between the colloids ${ }^{100}$. Based on excluded volume arguments, the indented colloids self-assemble into chains with a persistent length that depends of the depletant density. Explicit representation of depletants in simulations is not a trivial task due to the large size asymmetry between these particles and the large colloids in the system, and requires state-of-the-art Monte Carlo sampling algorithms. Quite remarkably, a similar system of indented colloids but with depletant modelled implicitly using an effective coarse-grained potential ${ }^{111,112}$ was found to also phase separate into two liquid phases: a depletantrich phase and a colloid-rich, porous phase characterised by the presence of polymeric colloidal aggregates ${ }^{111}$.

Crystallisation of indented colloids, however, still remains a challenge. Attempts to do so using, for example, sedimentation experiments have shown that these particles do not crystallise as easily as their spherical counterparts $^{113}$. More recently, the same behaviour was observed using computer simulation where it was demonstrated that the indentation frustrates the formation of hexagonal crystal monolayer phases typically observed in systems comprised of spherical particles of similar size ${ }^{114}$.

Lock-and-key complementary interactions are, of 
course, not exclusive to dimpled particles. In fact, recent studies have illustrated the limitless possibilities of using shape-complementarity to form structures of complex morphologies using particles with multiple indentations ${ }^{55,101,115}$. All the interesting behaviours found in lock-and-and key colloids has led to several follow-up simulation studies that have helped elucidate both the thermodynamics and kinetic mechanism of the associated phase transitions ${ }^{116-120}$.

FIG. 4. Representation of a lock and key colloidal systems. (a) Stacking of indented colloids; (b) typical dimerisation driven by lock and key interaction in a system of indented particle and spherical particles in the presence of depletants; (c) representative configuration of worm-like columns formed by stacking of bowl-like particles [Adapted from Ref. 54. Copyright 2010 American Chemical Society]; (d) self-assembled chain-like (polymeric) structures formed by lock and key interaction in a system of indented colloids [Adapted from Ref 100. Copyright 2013 Royal Society of Chemistry].

\section{Shape complementarity and size non-additive mixtures}

The behavior of colloidal systems with complementary shapes can be understood in terms of the well-known concept of size non-additive mixtures (NAM). In the case of binary mixtures of hard-spheres or Lennard-Jones beads of diameters $\sigma_{A}$ and $\sigma_{B}$, for example, size nonadditivity essentially implies that the cross-interaction van-der Waals repulsion parameter between $A$ and $B$ is $\sigma_{A B} \neq \frac{\left(\sigma_{A}+\sigma_{B}\right)}{2}$, so that, e.g., the so-called Lorentz combining rule is not obeyed and instead one writes $\sigma_{A B}=(1+\Delta) \frac{\left(\sigma_{A}+\sigma_{B}\right)}{2}$, where $\Delta=0$ for size additive mixtures and $\Delta \neq 0$ for non-additive mixtures (NAMs). NAMs have been of interest from both theoretical and practical perspective. Theoretically, NAMs provide a distinct non-energetic microscopic mechanism to induce complex macroscopic phase behaviors such as fluid-fluid phase separation; one of the earlier and extreme examples of NAM is the Widom-Rowlinson mixture ${ }^{94-96}$ where like species are non-interacting while unlike species experiences a hard-sphere potential (i.e., $\sigma_{A B}$ is finite while $\left.\sigma_{A A}=\sigma_{B B}=0\right)$. From a practical viewpoint, many real mixtures are known to have some degree of non-additivity which can lead to non-ideal mixture phase behavior. For example, non-additive hard spheres form compound solids that exhibit congruent melting/freezing ${ }^{97,98}$ (i.e., where the solid and liquid have the same composition), a behavior that is common in binary organic mixtures ${ }^{99}$.

In the context of NAMs, mixtures containing shape complementary components can be seen as cases where $\Delta<0$ even when the individual components may not have spherical shapes. Indeed, it is expected that qualitative features of the phase behavior of spherical NAMs would translate into non-spherical NAMs. One such key aspect is the effect of concentration or osmotic pressure on microstructure and phase behavior. This effect can be illustrated by means of a pseudo reaction that has associated a change in volume $\Delta V$ when the components $A$ and $B$ couple into a pseudo compound $A B$ :

$$
A+B \rightleftharpoons A B, \quad \Delta V
$$

In the case of bowl-like particles, for example, the sequential stacking of the particles can be expressed via the following pseudo-reactions with $\Delta V_{i}<0$ :

$$
\left[\begin{array}{cc}
A+A \rightleftharpoons A_{2}, & \Delta V_{1} \\
A+A_{2} \rightleftharpoons A_{3}, & \Delta V_{2} \\
\vdots \\
\text { etc }
\end{array}\right]
$$

In general, shape-complementary pairs have a $\Delta V<0$ which, in the case of spherical NAMs, correlates with $\Delta<0$. For non-convex particles $\Delta V$ would approximately quantify the entropic "patchiness" of the pair interactions. Equation 2 highlights the fact that the extent of inter-species coupling depends on the osmotic pressure $P$; i.e., the higher the $P$ the more to the right the equilibrium is shifted (toward the species with the smaller volume). Those cases where particles allow multiple simultaneous couplings so that polymer chains are assembled, can also be represented as additional pseudo reactions like:

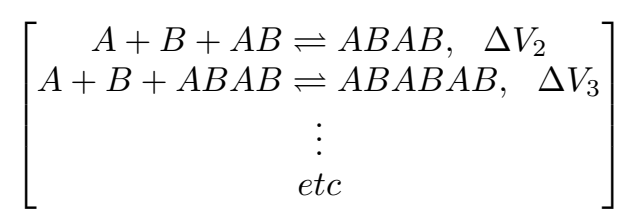

A key, often overlooked, point to realize is, however, that the transition from structures where the $A$ and $B$ type particles are predominantly uncoupled to structures where they are predominantly coupled is driven by a $P V$ contribution that lowers the free energy, i.e., by the fact that $P \Delta V<0$. Such an enthalpic $P V$ effect is activated, however, by virtue of the existence of readily accessible compact coupled configurations that pack space more efficiently (thus $\Delta V<0$ ), an effect that is often embodied by the idea of packing entropy. It is important to point out that the above analysis is also applicable to many pure-component and mixture systems of convex particles where a $\Delta V<0$ can be associated with the transition from a disordered to an ordered phase. However, shapecomplementary systems are especial not only in that the magnitude of $\Delta V<0$ is typically larger but also because that magnitude is intuitively easy to envision and control (via particle shape design). Future studies are expected to exploit such opportunities, likely in conjunction with depletion effects. 


\section{FINAL REMARKS}

As novel nano- and micro-sized building blocks, nonconvex particles offer limitless opportunities to fabricate new advanced materials. Recent experimental and simulation studies demonstrate the feasibility of using nonconvex geometries to engineer complex entropic interaction to drive the formation of such phases as porous ordered materials ${ }^{22,23}$, structures with either local or macroscopic chirality $40,47,121-123$, colloidal micelles ${ }^{50,71}$, etc. Evidently, there are several questions that have not yet been addressed and remain open to future investigations. For example, studies of nucleation kinetics in non-convex systems are required to have a better understanding of the bottlenecks hindering the formation of ordered structures ${ }^{59}$. Additional studies are also warranted to better understand how non-convex particles can reconfigure their shapes in response to their external environment, mimicking what certain biological systems do very efficiently ${ }^{26,124}$ Studies aimed to understand the collective behaviour and hydrodynamics of suspensions of non-convex particles are still in their infancy. Representative examples include studies of the dynamic behaviour of dilute suspension of branched particles ${ }^{125,126}$, selfpropelled C-shape particles ${ }^{39}$, non-convex multi-dimpled $\operatorname{discs}^{127,128}$, and spherical clusters ${ }^{129}$. Finally, theoretical models are needed to help explain and synthesise the complex phenomena arising in non-convex particles; while such studies have started to emerge $60,87,100,130$, there remain many challenges for the theoretical community.

\section{ACKNOWLEDGMENTS}

FAE acknowledges support from NSF award CBET1402117 .

${ }^{1}$ S. Sacanna and D. J. Pine, "Shape-anisotropic colloids: Building blocks for complex assemblies," Curr. Opin. Colloid Interface Sci. 16, 96-105 (2011).

$2 *$ This article reports a general experimental methodology to fabricate non-convex colloidal particles. S. Sacanna, M. Korpics, K. Rodriguez, L. Colon Melendez, S. H. Kim, D. J. Pine, and G. R. Yi, "Shaping colloids for self-assembly," Nat. Commun. 4, 1688 (2013).

${ }^{3}$ S. Sacanna, D. J. Pine, and G.-R. Yi, "Engineering shape: the novel geometries of colloidal self-assembly," Soft Matter 9, 8096-8106 (2013).

${ }^{4}$ V. Meester and D. J. Kraft, "Spherical, dimpled, and crumpled hybrid colloids with tunable surface morphology," Langmuir 32, 10668-10677 (2016)

${ }^{5}$ G. M. Whitesides and M. Boncheva, "Beyond molecules: Selfassembly of mesoscopic and macroscopic components," Proc. Natl. Acad. Sci. U. S. A. 99, 4769-4774 (2002).

${ }^{6}$ S. C. Glotzer and M. J. Solomon, "Anisotropy of building blocks and their assembly into complex structures," Nat. Mater. 6, 557-562 (2007).

${ }^{7}$ Q. Chen, S. C. Bae, and S. Granick, "Directed self-assembly of a colloidal kagome lattice," Nature 469, 381-384 (2011).

${ }^{8}$ G.-R. Yi, D. J. Pine, and S. Sacanna, "Recent progress on patchy colloids and their self-assembly," J. Phys. Condens. Matter 25, 193101 (2013).
${ }^{9}$ N. A. Mahynski, A. Z. Panagiotopoulos, D. Meng, and S. K. Kumar, "Stabilizing colloidal crystals by leveraging void distributions," Nat. Commun. 5, 4472 (2014).

${ }^{10}$ S. C. Glotzer, "Assembly engineering: Materials design for the 21st century (2013 PV Danckwerts lecture)," Chem. Eng. Sci. 121, 3-9 (2015).

${ }^{11} \mathrm{~L}$. Onsager, "The effects of shape on the interaction of colloidal particles," Ann. N. Y. Acad. Sci. 51, 627-659 (1949).

$12 *$ These three excellent articles discuss the role of entropy in colloidal systems. V. N. Manoharan, "Colloidal matter: Packing, geometry, and entropy," Science 349, 1253751 (2015); F. A. Escobedo, "Engineering entropy in soft matter: the bad, the ugly and the good," Soft Matter 10, 8388-8400 (2014); G. van Anders, D. Klotsa, N. K. Ahmed, M. Engel, and S. C. Glotzer, "Understanding shape entropy through local dense packing," Proc. Natl. Acad. Sci. U.S.A. 111, E4812-E4821 (2014).

${ }^{13}$ D. Frenkel, "Entropy-driven phase transitions," Physica A 263, 26-38 (1999).

${ }^{14}$ M. Dijkstra, "Entropy-driven phase transitions in colloids: From spheres to anisotropic particles," in Adv. Chem. Phys, Vol. 156 (John Wiley \& Sons, Inc., 2014) pp. 35-71.

${ }^{15}$ G. van Anders, N. K. Ahmed, R. Smith, M. Engel, and S. C. Glotzer, "Entropically patchy particles: engineering valence through shape entropy," ACS Nano 8, 931-940 (2014).

${ }^{16}$ P. F. Damasceno, M. Engel, and S. C. Glotzer, "Predictive selfassembly of polyhedra into complex structures," Science $\mathbf{3 3 7}$, 453-457 (2012).

${ }^{17}$ U. Agarwal and F. A. Escobedo, "Mesophase behaviour of polyhedral particles," Nat. Mater. 10, 230-235 (2011).

${ }^{18}$ W. L. Miller and A. Cacciuto, "On the phase behavior of hard aspherical particles," J. Chem. Phys. 133, 234903 (2010).

${ }^{19}$ R. Memmer, "Liquid crystal phases of achiral banana-shaped molecules: a computer simulation study," Liq. Cryst. 29, 483496 (2002).

${ }^{20}$ M. Hird, "Banana-shaped and other bent-core liquid crystals," Liq. Cryst. Today 14, 9-21 (2005).

${ }^{21}$ R. Gabbrielli, Y. Jiao, and S. Torquato, "Dense periodic packings of tori," Phys. Rev. E 89, 022133 (2014).

$22 *$ A unique example of a porous liquid crystals driven by interlocking effects. C. Avendaño, G. Jackson, E. A. Müller, and F. A. Escobedo, "Assembly of porous smectic structures formed from interlocking high-symmetry planar nanorings," Proc. Natl. Acad. Sci. U. S. A. 113, 9699-9703 (2016).

$23 *$ Example of the formation of porous colloidal crystals driven by interlocking effects. K. Miszta, J. de Graaf, G. Bertoni, D. Dorfs, R. Brescia, S. Marras, L. Ceseracciu, R. Cingolani, R. van Roij, M. Dijkstra, and L. Manna, "Hierarchical self-assembly of suspended branched colloidal nanocrystals into superlattice structures," Nat. Mater. 10, 872-876 (2011).

$24 *$ This article reports a large variety of closed-packed structure observed in particles with non-convex geometries. J. de Graaf, R. van Roij, and M. Dijkstra, "Dense regular packings of irregular nonconvex particles," Phys. Rev. Lett. 107, 155501 (2011).

${ }^{25}$ S. Atkinson, Y. Jiao, and S. Torquato, "Maximally dense packings of two-dimensional convex and concave noncircular particles," Phys. Rev. E 86, 031302 (2012).

${ }^{26}$ T. D. Nguyen, E. Jankowski, and S. C. Glotzer, "Self-assembly and reconfigurability of shape-shifting particles," ACS Nano $\mathbf{5}$, 8892-8903 (2011).

${ }^{27}$ N. Gravish, S. V. Franklin, D. L. Hu, and D. I. Goldman, "Entangled granular media," Phys. Rev. Lett. 108, 208001 (2012).

${ }^{28}$ H. Jaeger, "Toward jamming by design," Soft Matter 11, 12 (2015).

${ }^{29}$ C. Hoell and H. Löwen, "Colloidal suspensions of C-particles: Entanglement, percolation and microrheology," J. Chem. Phys. 144, 174901 (2016).

${ }^{30}$ S. Ungphaiboon, D. Attia, G. G. D'Ayala, P. Sansongsak, F. Cellesi, and N. Tirelli, "Materials for microencapsulation: what toroidal particles doughnuts" can do better than spherical beads," Soft Matter 6, 4070 (2010). 
${ }^{31}$ B. Pang, Y. Zhao, H. Luehmann, X. Yang, L. Detering, M. You, C. Zhang, L. Zhang, Z.-Y. Li, Q. Ren, Y. Liu, and Y. Xia, "Cu-64-Doped PdCu@Au Tripods: A Multifunctional Nanomaterial for Positron Emission Tomography and Image-Guided Photothermal Cancer Treatment," ACS Nano 10, 3121-3131 (2016).

${ }^{32}$ C. Chen, Y. Kang, Z. Huo, Z. Zhu, W. Huang, H. L. Xin, J. D. Snyder, D. Li, J. A. Herron, M. Mavrikakis, M. Chi, K. L. More, Y. Li, N. M. Markovic, G. A. Somorjai, P. Yang, and V. R. Stamenkovic, "Highly crystalline multimetallic nanoframes with three-dimensional electrocatalytic surfaces," Science 343, 1339 (2014).

${ }^{33}$ X. Kong, Y. Ding, R. Yang, and Z. Wang, "Single-crystal nanorings formed by epitaxial self-coiling of polar nanobelts," Science 303, 1348-1351 (2004).

${ }^{34}$ I. D. Hosein, M. Ghebrebrhan, J. D. Joannopoulos, and C. M. Liddell, "Dimer shape anisotropy: A nonspherical colloidal approach to omnidirectonal photonic band gaps," Langmuir $\mathbf{2 6}$ 2151-2159 (2010).

${ }^{35}$ Z. Cheng, F. Luo, Z. Zhang, and Y.-Q. Ma, "Syntheses and applications of concave and convex colloids with precisely controlled shapes," Soft Matter 9, 11392-11397 (2013).

$36 *$ This experimental paper demonstrates the role of nonconvexity in the formation of porous liquids. N. Giri, M. G. Del Popolo, G. Melaugh, R. L. Greenaway, K. Raetzke, T. Koschine, L. Pison, M. F. C. Gomes, A. I. Cooper, and S. L. James "Liquids with permanent porosity," Nature 527, 216 (2015).

${ }^{37}$ L. R. Meza, S. Das, and J. R. Greer, "Strong, lightweight, and recoverable three-dimensional ceramic nanolattices," Science 345, 1322-1326 (2014)

${ }^{38}$ W. Qi, J. de Graaf, F. Qiao, S. Marras, L. Manna, and M. Dijkstra, "Ordered two-dimensional superstructures of colloidal octapod-shaped nanocrystals on flat substrates," Nano Lett. 12, 5299-5303 (2012).

${ }^{39}$ H. H. Wensink, V. Kantsler, R. E. Goldstein, and J. Dunkel, "Controlling active self-assembly through broken particle-shape symmetry," Phys. Rev. E 89, 010302 (2014).

${ }^{40}$ P. Y. Wang and T. G. Mason, "Colloidal lock-and-key dimerization reactions of hard annular sector particles controlled by osmotic pressure," J. Am. Chem. Soc. 137, 15308-15314 (2015).

${ }^{41}$ B. Senyuk, M. B. Pandey, Q. Liu, M. Tasinkevych, and I. I. Smalyukh, "Colloidal spirals in nematic liquid crystals," Soft Matter 11, 8758-8767 (2015).

${ }^{42}$ M. S. Yavuz, Y. Cheng, J. Chen, C. M. Cobley, Q. Zhang, M. Rycenga, J. Xie, C. Kim, K. H. Song, A. G. Schwartz, L. V. Wang, and Y. Xia, "Gold nanocages covered by smart polymers for controlled release with near-infrared light," Nat. Mater. 8, 935-939 (2009).

${ }^{43}$ A. Martinez, M. Ravnik, B. Lucero, R. Visvanathan, S. Zumer, and I. I. Smalyukh, "Mutually tangled colloidal knots and induced defect loops in nematic fields," Nat. Mater. 13, 259-264 (2014).

${ }^{44}$ Y. Tian, T. Wang, W. Liu, H. L. Xin, H. Li, Y. Ke, W. M. Shih, and O. Gang, "Prescribed nanoparticle cluster architectures and low-dimensional arrays built using octahedral DNA origami frames," Nat. Nanotechnol. 10, 637 (2015).

${ }^{45}$ T. Paik and C. B. Murray, "Shape-directed binary assembly of anisotropic nanoplates: A nanocrystal puzzle with shapecomplementary building blocks," Nano Lett. 13, 2952-2956 (2013).

46 M. P. Arciniegas, M. R. Kim, J. de Graaf, R. Brescia, S. Marras, K. Miszta, M. Dijkstra, R. van Roij, and L. Manna, "Selfassembly of octapod-shaped colloidal nanocrystals into a hexagonal ballerina network embedded in a thin polymer film," Nano Lett. 14, 1056-1063 (2014).

${ }^{47}$ K. Zhao and T. G. Mason, "Self-organized chiral colloidal crystals of brownian square crosses," J. Phys. Condens. Matter 26, 152101 (2014).

${ }^{48}$ H. Zhang, Y. Lu, H. Liu, and J. Fang, "One-pot synthesis of high-index faceted $\mathrm{AgCl}$ nanocrystals with trapezohedral, con- cave hexoctahedral structures and their photocatalytic activity," Nanoscale 7, 11591-11601 (2015).

${ }^{49}$ J.-W. Kim, R. J. Larsen, and D. A. Weitz, "Uniform nonspherical colloidal particles with tunable shapes," Adv. Mater. 19, 2005 (2007).

${ }^{50}$ J. R. Wolters, G. Avvisati, F. Hagemans, T. Vissers, D. J. Kraft, M. Dijkstra, and W. K. Kegel, "Self-assembly of "mickey mouse" shaped colloids into tube-like structures: experiments and simulations," Soft Matter 11, 1067-1077 (2015).

${ }^{51}$ C. I. Zoldesi, C. A. van Walree, and A. Imhof, "Deformable hollow hybrid silica/siloxane colloids by emulsion templating," Langmuir 22, 4343-4352 (2006).

52 I. D. Hosein and C. M. Liddell, "Convectively assembled nonspherical mushroom cap-based colloidal crystals," Langmuir 23, 8810-8814 (2007).

$53 * *$ This experimental paper demonstrates the concept of shape complementarity in colloidal particles. S. Sacanna, W. T. M. Irvine, P. M. Chaikin, and D. J. Pine, "Lock and key colloids," Nature 464, 575-578 (2010).

$54 *$ This computer simulation study shows the formation of stacking in colloidal bowls .M. Marechal, R. J. Kortschot, A. F. Demirors, A. Imhof, and M. Dijkstra, "Phase behavior and structure of a new colloidal model system of bowl-shaped particles," Nano Lett. 10, 1907-1911 (2010).

${ }^{55}$ Y. Wang, Y. Wang, X. Zheng, G.-R. Yi, S. Sacanna, D. J. Pine, and M. Weck, "Three-dimensional lock and key colloids," J. Am. Chem. Soc. 136, 6866-6869 (2014).

${ }^{56} \mathrm{~K}$. Zhao and T. G. Mason, "Directing colloidal self-assembly through roughness-controlled depletion attractions," Phys. Rev. Lett. 99, 268301 (2007).

${ }^{57}$ J. D. Forster, J.-G. Park, M. Mittal, H. Noh, C. F. Schreck, C. S. O'Hern, H. Cao, E. M. Furst, and E. R. Dufresne, "Assembly of optical-scale dumbbells into dense photonic crystals," ACS Nano 5, 6695-6700 (2011).

${ }^{58}$ M. Marechal and M. Dijkstra, "Stability of orientationally disordered crystal structures of colloidal hard dumbbells," Phys. Rev. E 77, 061405 (2008).

${ }^{59} \mathrm{R}$. Ni and M. Dijkstra, "Crystal nucleation of colloidal hard dumbbells," J. Chem. Phys. 134, 034501 (2011)

${ }^{60}$ M. Marechal, H. H. Goetzke, A. Haertel, and H. Löwen, "Inhomogeneous fluids of colloidal hard dumbbells: Fundamental measure theory and monte carlo simulations," J. Chem. Phys. 135, 234510 (2011).

${ }^{61}$ M. Dennison, K. Milinkovic, and M. Dijkstra, "Phase diagram of hard snowman-shaped particles," J. Chem. Phys. 137, 044507 (2012).

${ }^{62}$ K. Milinkovic, M. Dennison, and M. Dijkstra, "Phase diagram of hard asymmetric dumbbell particles," Phys. Rev. E 87, 032128 (2013)

${ }^{63}$ K. W. Wojciechowski, D. Frenkel, and A. C. Brańka, "Nonperiodic solid phase in a two-dimensional hard-dimer system," Phys. Rev. Lett. 66, 3168-3171 (1991).

${ }^{64}$ C. Vega, E. P. A. Paras, and P. A. Monson, "Solid-fluid equilibria for hard dumbbells via monte carlo simulation," J. Chem. Phys. 96, 9060-9072 (1992)

${ }^{65}$ S. H. Lee, S. J. Gerbode, B. S. John, A. K. Wolfgang, F. A. Escobedo, I. Cohen, and C. M. Liddell, "Synthesis and assembly of nonspherical hollow silica colloids under confinement," J. Mater. Chem. , 4912-4916 (2008).

${ }^{66}$ K. Muangnapoh, C. Avendaño, F. A. Escobedo, and C. M. Liddell-Watson, "Degenerate crystals from colloidal dimers under confinement," Soft Matter 10, 9729-9738 (2014).

${ }^{67}$ S. J. Gerbode, S. H. Lee, C. M. Liddell, and I. Cohen, "Restricted dislocation motion in crystals of colloidal dimer particles," Phys. Rev. Lett. 101, 058302 (2008).

$68 *$ This article study the dynamics of dislocation in a crystal of colloidal dimers. S. J. Gerbode, U. Agarwal, D. C. Ong, C. M. Liddell, F. A. Escobedo, and I. Cohen, "Glassy dislocation dynamics in 2D colloidal dimer crystals," Phys. Rev. Lett. 105, 078301 (2010) 
${ }^{69}$ U. Agarwal and F. A. Escobedo, "Yielding and shear induced melting of 2D mixed crystals of spheres and dimers," Soft Matter 8, 5916-5922 (2012).

${ }^{70}$ C. Jennings, M. Ramsay, T. Hudson, and P. Harrowell, "Packing concave molecules in crystals and amorphous solids: on the connection between shape and local structure," Mol. Phys. 113, 2755-2769 (2015)

$71 *$ This article shows the formation of colloidal micelles through the use of surface roughness. D. J. Kraft, R. Ni, F. Smallenburg, M. Hermes, K. Yoon, D. A. Weitz, A. van Blaaderen, J. Groenewold, J. Dijkstra, and W. K. Kegel, "Surface roughness directed self-assembly of patchy particles into colloidal micelles," Proc. Natl. Acad. Sci. U.S.A. 109, 10787-10792 (2012).

${ }^{72}$ S. Jiang, Q. Chen, M. Tripathy, E. Luijten, K. S. Schweizer, and S. Granick, "Janus particle synthesis and assembly," Adv. Mater. 22, 1060-1071 (2010).

${ }^{73}$ D. J. Kraft, J. Hilhorst, M. A. P. Heinen, M. J. Hoogenraad, B. Luigjes, and W. K. Kegel, "Patchy polymer colloids with tunable anisotropy dimensions," J. Phys. Chem. B 115, 71757181 (2011).

${ }^{74}$ J.-G. Park, J. D. Forster, and E. R. Dufresne, "Synthesis of colloidal particles with the symmetry of water molecules," Langmuir 25, 8903-8906 (2009).

${ }^{75}$ V. Meester, R. W. Verweij, C. van der Wel, and D. J. Kraft, "Colloidal recycling: Reconfiguration of random aggregates into patchy particles," ACS Nano 10, 4322-4329 (2016).

${ }^{76}$ W. L. Miller, B. Bozorgui, and A. Cacciuto, "Crystallization of hard aspherical particles," J. Chem. Phys. 132, 134901 (2010).

${ }^{77}$ A. Gruenberger, P.-K. Lai, M. A. Blanco, and C. J. Roberts, "Coarse-grained modeling of protein second osmotic virial coefficients: Sterics and short-ranged attractions," J. Phys. Chem. B 117, 763-770 (2013).

${ }^{78}$ C. Calero-Rubio, A. Saluja, and C. J. Roberts, "Coarse-grained antibody models for "weak" protein-protein interactions from low to high concentrations," J. Phys. Chem. B 120, 6592-6605 (2016).

${ }^{79}$ S. Torquato and F. H. Stillinger, "Jammed hard-particle packings: From Kepler to Bernal and beyond," Rev. Mod. Phys. 82, 2633-2672 (2010)

${ }^{80}$ N. Gravish, S. V. Franklin, D. L. Hu, and D. I. Goldman, "Entangled granular media," Phys. Rev. Lett. 108, 208001 (2012).

${ }^{81} \mathrm{~S}$. Torquato and Y. Jiao, "Organizing principles for dense packings of nonspherical hard particles: Not all shapes are created equal," Phys. Rev. E 86, 011102 (2012).

${ }^{82}$ F. Ludewig and N. Vandewalle, "Strong interlocking of nonconvex particles in random packings," Phys. Rev. E 85, 051307 (2012).

${ }^{83}$ E. Gonzalez, J. Arbiol, and V. F. Puntes, "Carving at the nanoscale: Sequential galvanic exchange and kirkendall growth at room temperature," Science 334, 1377-1380 (2011).

${ }^{84}$ R. Veneziano, S. Ratanalert, K. Zhang, F. Zhang, H. Yan, W. Chiu, and M. Bathe, "Designer nanoscale DNA assemblies programmed from the top down," Science 352, 1534 (2016).

${ }^{85}$ C. Avendaño and E. A. Müller, "Liquid crystalline and antinematic behavior of shape-persistent macrocycles from moleculardynamics simulations," Phys. Rev. E 80, 061702 (2009).

${ }^{86} \mathrm{C}$. Avendaño and E. A. Müller, "Liquid crystalline behavior of a coarse-grained model of shape-persistent macrocycles with flexible attractive chains," Soft Matter 7, 1694-1701 (2011).

${ }^{87}$ H. H. Wensink and C. Avendaño, "Empty smectic liquid crystals of hard nanorings: Insights from a second-virial theory," Phys. Rev. E 94, 062704 (2016).

${ }^{88}$ B. Lim, M. Jiang, P. H. C. Camargo, E. C. Cho, J. Tao, X. Lu Y. Zhu, and Y. Xia, "Pd-Pt bimetallic nanodendrites with high activity for oxygen reduction," Science 324, 1302-1305 (2009).

${ }^{89}$ H. Zhang, D.-H. Ha, R. Hovden, L. F. Kourkoutis, and R. D. Robinson, "Controlled synthesis of uniform cobalt phosphide hyperbranched nanocrystals using tri-n-octylphosphine oxide as a phosphorus source," Nano Lett. 11, 188-197 (2011).
${ }^{90}$ D. W. Sinkovits and E. Luijten, "Nanoparticle-controlled aggregation of colloidal tetrapods," Nano Lett. 12, 1743-1748 (2012).

${ }^{91}$ H. Li, A. G. Kanaras, and L. Manna, "Colloidal branched semiconductor nanocrystals: State of the art and perspectives," Acc. Chem. Res. 46, 1387-1396 (2013).

${ }^{92}$ W. Qi, J. de Graaf, F. Qiao, S. Marras, L. Manna, and M. Dijkstra, "Phase diagram of octapod-shaped nanocrystals in a quasitwo-dimensional planar geometry," J. Chem. Phys. 138, 154504 (2013).

93 A. Castelli, J. de Graaf, M. Prato, L. Manna, and M. P. Arciniegas, "Tic-tac-toe binary lattices from the interfacial selfassembly of branched and spherical nanocrystals," ACS Nano 10, 4345-4353 (2016).

${ }^{94} \mathrm{~B}$. Widom and J. S. Rowlinson, "New model for the study of liquid-vapor phase transitions," J. Chem. Phys. 52, 1670-1684 (1970).

${ }^{95}$ C.-Y. Shew and A. Yethiraj, "Phase behavior of the widomrowlinson mixture," J. Chem. Phys. 104, 7665-7670 (1996).

${ }^{96}$ W. T. Góźdź, "Phase separation of the Widom-Rowlinson mixture in confined geometry," J. Chem. Phys. 122, 074505 (2005).

${ }^{97}$ M. J. Vlot, J. C. van Miltenburg, H. A. J. Oonk, and J. P. van der Eerden, "Phase diagrams of scalemic mixtures: A Monte Carlo simulation study," J. Chem. Phys. 107, 10102-10111 (1997).

${ }^{8}$ S. Punnathanam and P. A. Monson, "Crystal nucleation in binary hard sphere mixtures: A monte carlo simulation study," J. Chem. Phys. 125, 024508 (2006).

${ }^{99}$ M. Matsuoka, "Solid liquid equilibria of binary organic mixtures," Bunri Gijutsu (Separation Process Engineering) 7, 245249 (1977).

${ }^{100}$ D. J. Ashton, R. L. Jack, and N. B. Wilding, "Self-assembly of colloidal polymers via depletion-mediated lock and key binding," Soft Matter 9, 9661-9666 (2013).

${ }^{101}$ N. K. Ahmed, G. van Anders, E. R. Chen, and S. C. Glotzer, "Crossover behavior in the packing and assembly of multivalent lock-and-key colloids," arXiv preprint arXiv:1501.03130 (2016).

${ }^{102}$ M. Marechal and M. Dijkstra, "Phase behavior and structure of colloidal bowl-shaped particles: Simulations," Phys. Rev. E 82, 031405 (2010)

${ }^{103}$ B. Xu and T. M. Swager, "Rigid bowlic liquid crystals based on tungsten-oxo calix [4] arenes: host-guest effects and head-to-tail organization," J. Am. Chem. Soc. 115, 1159-1160 (1993).

${ }^{104}$ M. Sawamura, K. Kawai, Y. Matsuo, K. Kanie, T. Kato, and E. Nakamura, "Stacking of conical molecules with a fullerene apex into polar columns in crystals and liquid crystals," Nature 419, 702-705 (2002).

${ }^{105}$ G. Cinacchi and J. S. van Duijneveldt, "Phase behavior of contact lens-like particles: Entropy-driven competition between isotropic- nematic phase separation and clustering," J. Phys. Chem. Lett. 1, 787-791 (2010).

${ }^{106}$ G. Cinacchi, "Phase behavior of hard spherical caps," J. Chem. Phys. 139, 124908 (2013).

${ }^{107}$ L. Filion, M. Marechal, B. van Oorschot, D. Pelt, F. Smallenburg, and M. Dijkstra, "Efficient method for predicting crystal structures at finite temperature: Variable box shape simulations," Phys. Rev. Lett. 103, 188302 (2009).

${ }^{108}$ C. Avendaño, C. M. Liddell-Watson, and F. A. Escobedo, "Directed self-assembly of spherical caps via confinement," Soft Matter 9, 9153-9166 (2013).

${ }^{109}$ J. Mcbride and C. Avendaño, "Phase behaviour and gravitydirected self assembly of hard convex spherical caps," Soft Matter 13, 2085-2098 (2017).

${ }^{110}$ S. Sacanna, W. T. Irvine, L. Rossi, and D. J. Pine, "Lock and key colloids through polymerization-induced buckling of monodisperse silicon oil droplets," Soft Matter 7, 1631-1634 (2011).

$111 *$ This simulation study shows the formation of porous colloidal liquids. D. J. Ashton, R. L. Jack, and N. B. Wilding, "Porous liquid phases for indented colloids with depletion interactions," Phys. Rev. Lett. 114, 237801 (2015). 
${ }^{112}$ C. Law, D. J. Ashton, N. B. Wilding, and R. L. Jack, "Coarsegrained depletion potentials for anisotropic colloids: Application to lock-and-key systems," J. Chem. Phys. 145, 084907 (2016).

${ }^{113}$ S. J. Ivell, R. P. A. Dullens, S. Sacanna, and D. G. A. L. Aarts, "Emerging structural disorder in a suspension of uniformly dimpled colloidal particles," Soft Matter 9, 9361-9365 (2013).

${ }^{114}$ D. J. Ashton, S. J. Ivell, R. P. Dullens, R. L. Jack, N. B. Wilding, and D. G. Aarts, "Self-assembly and crystallisation of indented colloids at a planar wall," Soft Matter 11, 6089-6098 (2015).

${ }^{115}$ E. S. Harper, R. L. Marson, J. A. Anderson, G. Van Anders, and S. C. Glotzer, "Shape allophiles improve entropic assembly," Soft Matter 11, 7250-7256 (2015).

${ }^{116} \mathrm{G}$. Odriozola and M. Lozada-Cassou, "Statistical mechanics approach to lock-key supramolecular chemistry interactions," Phys. Rev. Lett. 110, 105701 (2013).

${ }^{117}$ G. Odriozola and M. Lozada-Cassou, "Entropy driven selfassembly in charged lock-key particles," J. Phys. Chem. B 120, 5966-5974 (2016).

${ }^{118}$ L. Colon-Melendez, D. J. Beltran-Villegas, G. van Anders, J. Liu, M. Spellings, S. Sacanna, D. J. Pine, S. C. Glotzer, R. G. Larson, and M. J. Solomon, "Binding kinetics of lock and key colloids," J. Chem. Phys. 142, 174909 (2015).

${ }^{119}$ D. J. Beltran-Villegas, L. Colon-Melendez, M. J. Solomon, and R. G. Larson, "Kinetic modeling and design of colloidal lock and key assembly," J. Colloid Interface Sci. 463, 242-257 (2016)

${ }^{120}$ H.-Y. Chang, C.-W. Huang, Y.-F. Chen, S.-Y. Chen, Y.-J. Sheng, and H.-K. Tsao, "Assembly of lock-and-key colloids mediated by polymeric depletant," Langmuir 31, 13085-13093 (2015).

${ }^{121}$ S. Dussi and M. Dijkstra, "Entropy-driven formation of chiral nematic phases by computer simulations," Nat. Commun. 7,
11175 (2016).

${ }^{122}$ H. B. Kolli, G. Cinacchi, A. Ferrarini, and A. Giacometti, "Chiral self-assembly of helical particles," Faraday Discuss. 186 171-186 (2016).

${ }^{123}$ P.-Y. Wang and T. G. Mason, "Dimer crystallization of chiral proteoids," Phys. Chem. Chem. Phys. 19, 7167-7175 (2017).

${ }^{124}$ M. Youssef, T. Hueckel, G.-R. Yi, and S. Sacanna, "Shapeshifting colloids via stimulated dewetting," Nat. Commun. 7, 12216 (2016).

${ }^{125}$ M. K. Petersen, J. M. D. Lane, and G. S. Grest, "Shear rheology of extended nanoparticles," Phys. Rev. Ehys 82, 010201 (2010).

${ }^{126}$ D. R. Heine, M. K. Petersen, and G. S. Grest, "Effect of particle shape and charge on bulk rheology of nanoparticle suspensions," J. Chem. Phys. 132, 184509 (2010).

${ }^{127}$ N. H. Nguyen, D. Klotsa, M. Engel, and S. C. Glotzer, "Emergent collective phenomena in a mixture of hard shapes through active rotation," Phys. Rev. Lett. 112, 075701 (2014).

${ }^{128}$ M. Spellings, M. Engel, D. Klotsa, S. Sabrina, A. M. Drews, N. H. Nguyen, K. J. Bishop, and S. C. Glotzer, "Shape control and compartmentalization in active colloidal cells," Proc. Natl. Acad. Sci. U.S.A. 112, E4642-E4650 (2015).

${ }^{129}$ D. J. Kraft, R. Wittkowski, B. Ten Hagen, K. V. Edmond, D. J. Pine, and H. Löwen, "Brownian motion and the hydrodynamic friction tensor for colloidal particles of complex shape," Phys. Rev. E 88, 050301 (2013).

${ }^{130}$ W. D. Hodson and T. G. Mason, "Lock-and-key dimerization in dense brownian systems of hard annular sector particles," Phys. Rev. E 94, 022124 (2016). 
(a)

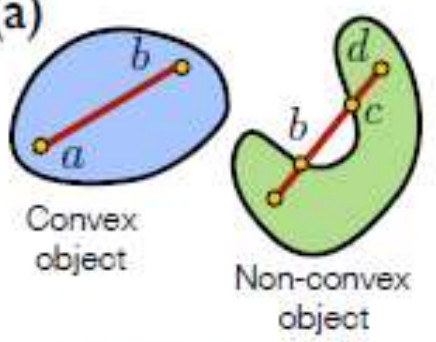

(d)
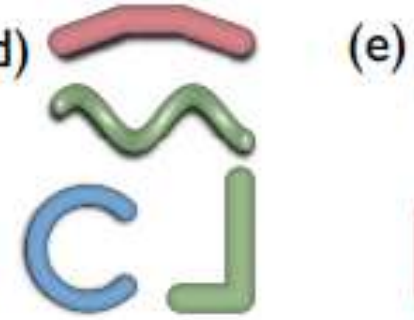

Curved, bent and twisted particles

(e) (b)
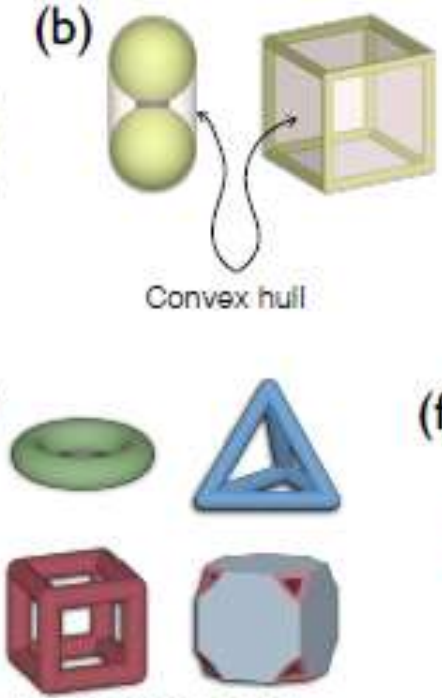

Rings, frames and cages (c)

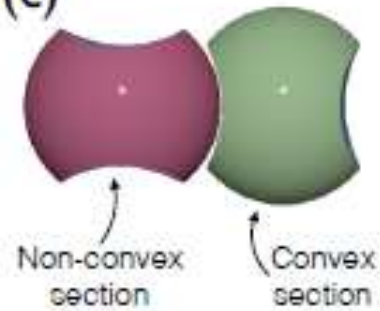

(f)

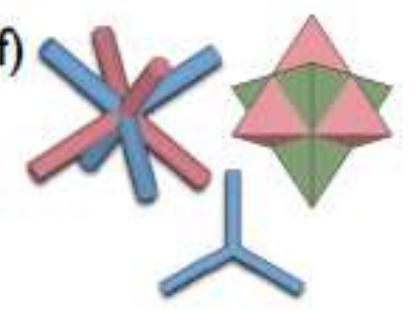

Branched particles and non-convex polyhedra (g)

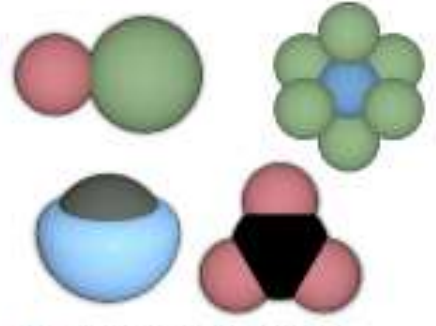

Dimers, protrusions, and spherical clusters (h)
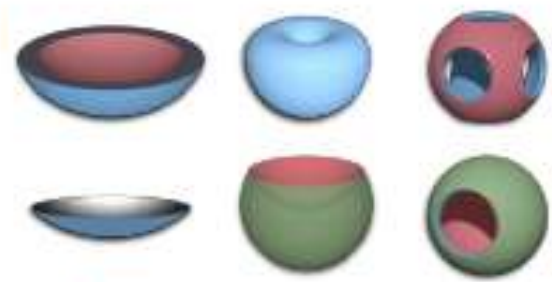

Bowls, contact lenses, and indented colloids

Figure 1 

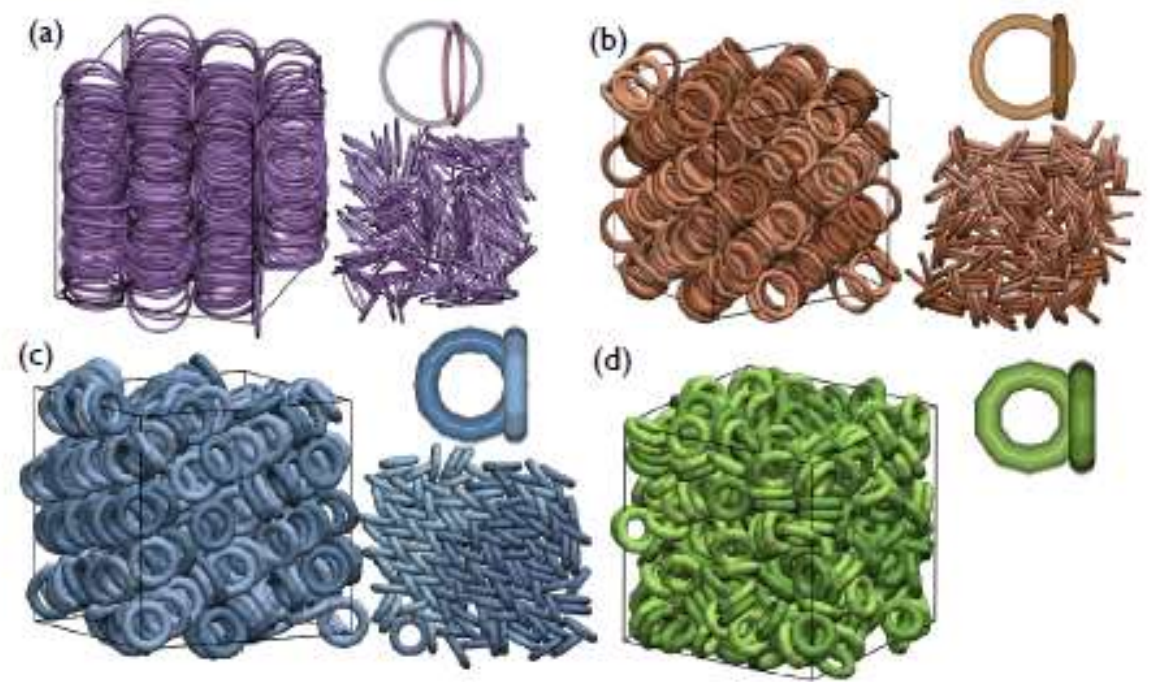

Figure 2

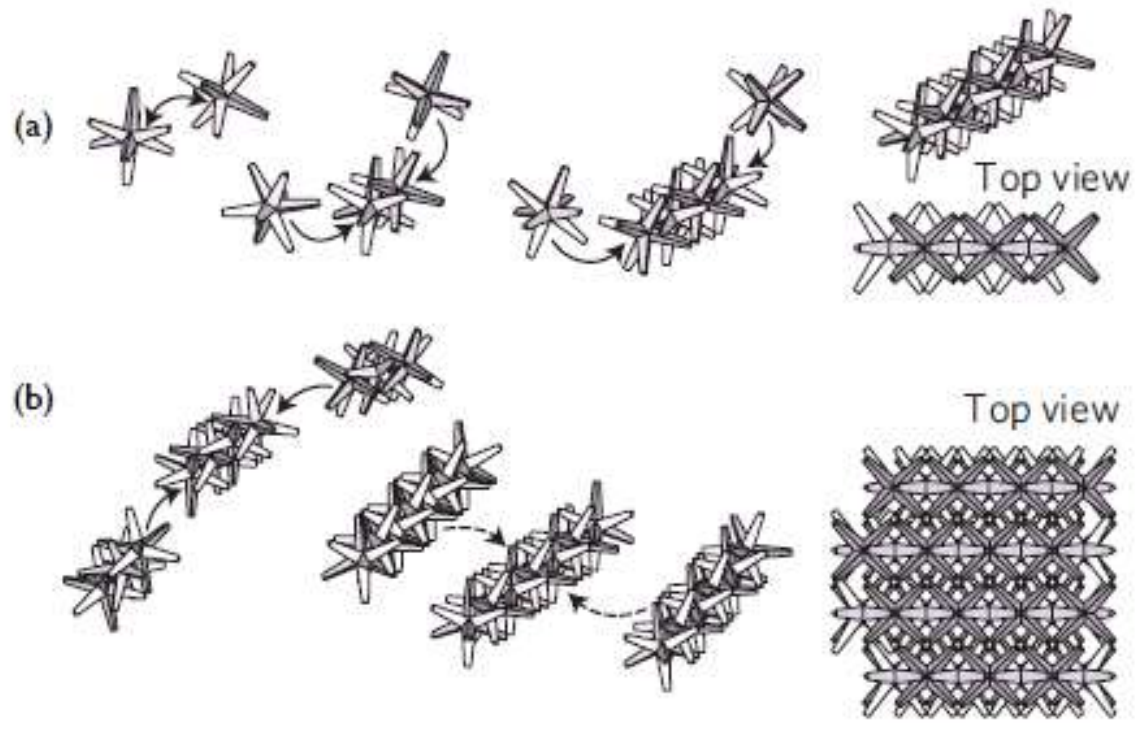

Figure 3 
(a)
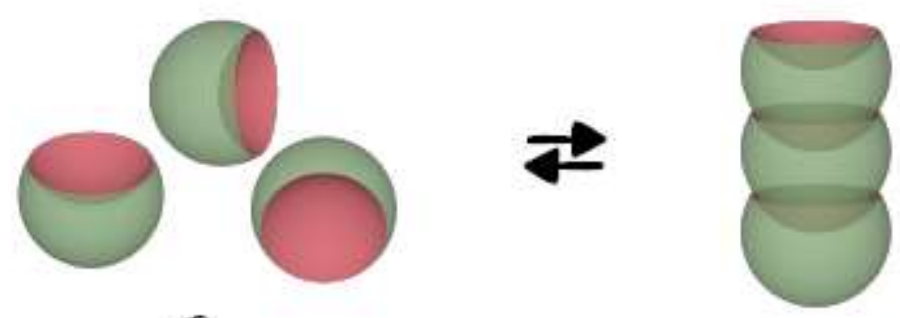

(b)
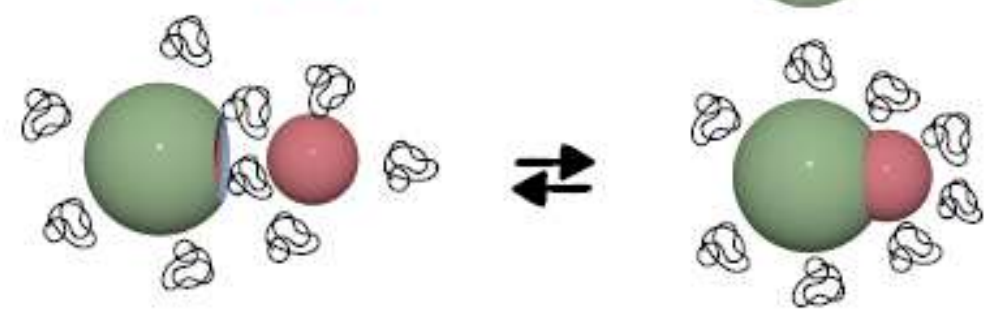

(c)

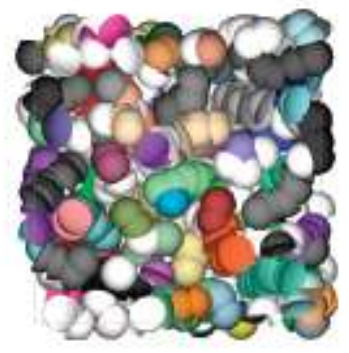

(d)

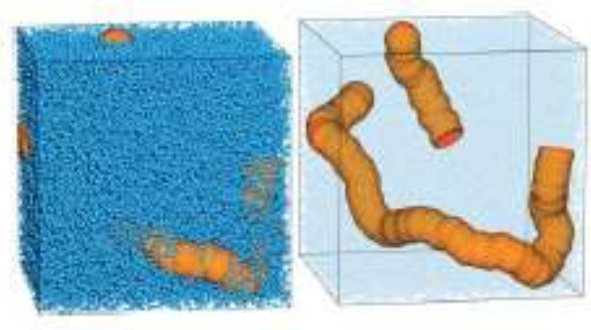

Figure 4 
Graphical abstract

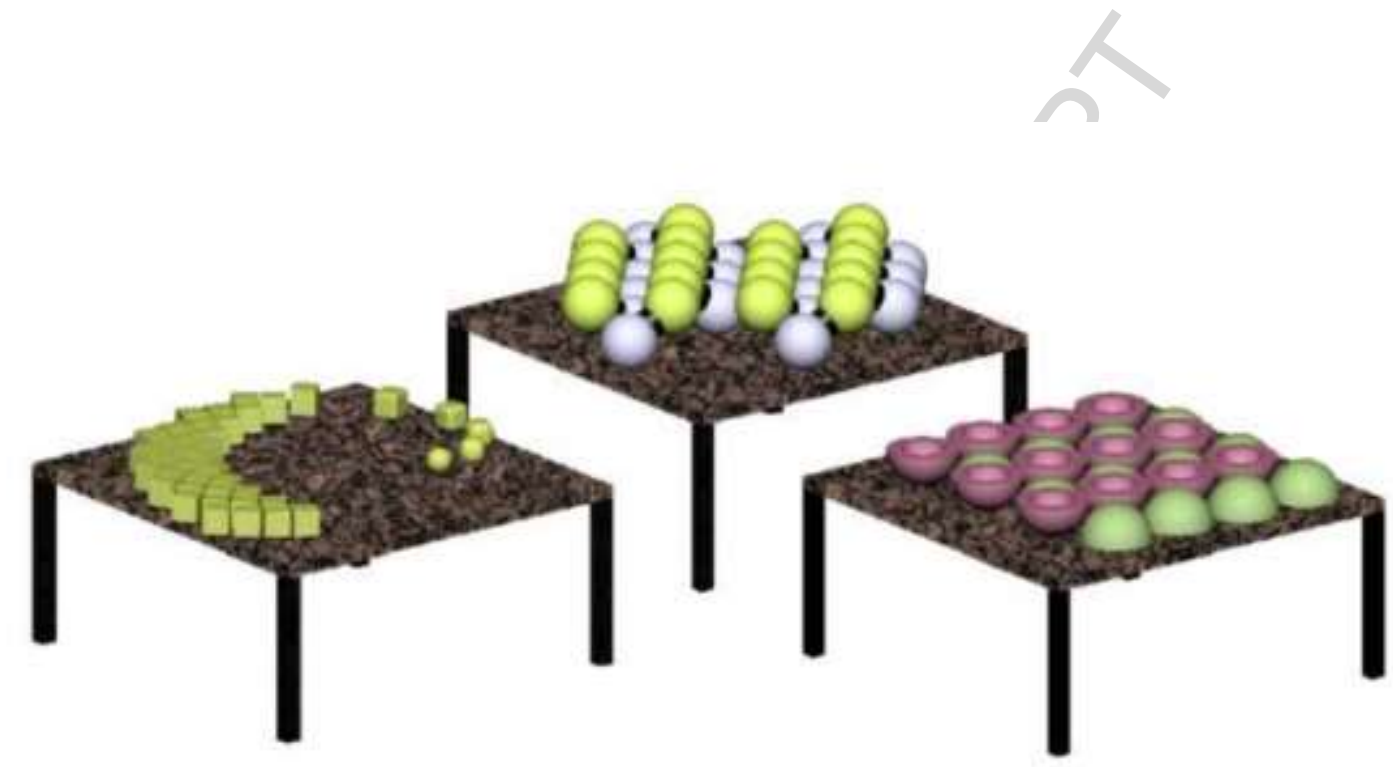

